\title{
Development of Engineering Model Providing Body Force Distribution of Tri-Electrode Plasma Actuator*
}

\author{
Kumi NAKaI, ${ }^{1{ }^{\dagger} \dagger}$ Hiroyuki NishidA, ${ }^{1)}$ Norio AsaumI ${ }^{2)}$ Yuhi TANAKA,${ }^{2)}$ and Takashi MatsunO ${ }^{3)}$ \\ ${ }^{1)}$ Department of Mechanical Systems Engineering, Tokyo University of Agriculture and Technology, Koganei, Tokyo 184-8588, Japan \\ ${ }^{2)}$ Research Laboratory, IHI Corporation, Yokohama, Kanagawa 235-8501, Japan \\ ${ }^{3)}$ Department of Mechanical and Aerospace Engineering, Tottori University, Tottori, Tottori 680-8552, Japan
}

\begin{abstract}
Tri-electrode plasma actuators (TED-PAs) can induce a stronger jet than that of conventional two-electrode plasma actuators (DBDPAs). For practical application of a TED-PA, it is significant to develop a TED-PA engineering model for implementing CFD simulations. In this study, we model the body force distribution generated by a TED-PA utilizing the Suzen model, which is one of engineering models used for DBDPAs. First, we define a function to describe the charge distribution profile on the dielectric surface with two half-Gaussian distributions. Second, we define the maximum values of surface charge as functions of the voltage applied based on the plasma simulation results. Finally, the flow fields numerically obtained using the model developed are compared with the experimental results from our previous study. Although there are some discrepancies mainly due to the two-dimensional laminar flow simulation, the model developed can quantitatively reproduce the voltage characteristics of thrust force and the jet structure induced. Therefore, the model developed is expected to evaluate the flow control effect precisely.
\end{abstract}

Key Words: Plasma Actuator, Body Force, Engineering Model, Computational Fluid Dynamics

\author{
Nomenclature \\ $\boldsymbol{E}$ : electric field vector \\ $\varepsilon_{r}:$ relative electric permittivity \\ $\boldsymbol{F}$ : body force vector \\ $\lambda_{D}$ : Debye length \\ $\omega$ : frequency \\ $\phi$ : electric potential \\ $\rho_{c}$ : charge density \\ $t$ : time \\ $V$ : voltage \\ $x$ : coordinate \\ Subscripts \\ AC: AC electrode \\ DC: DC electrode
}

\section{Introduction}

Dielectric barrier discharge plasma actuators (DBDPAs) are flow control devices that utilize a dielectric barrier discharge, and are attracting much attention. ${ }^{1-5)}$ The typical configuration of a DBDPA is shown in Fig. 1(a). It consists of two electrodes (air-exposed electrode and covered electrode) separated by a dielectric. When a high-voltage alternating current (typically, several $\mathrm{kHz}$ and several $\mathrm{kV}$ ) is applied between the electrodes, DBD occurs and the body force acting on the ambient gas is generated by the collisions

(C) 2019 The Japan Society for Aeronautical and Space Sciences

*Presented at the 31st International Symposium on Space Technology and Science, 3-9 June 2017, Matsuyama, Japan.

Received 4 December 2017; final revision received 23 February 2019; accepted for publication 19 March 2019.

†Corresponding author, kumi-nakai@st.go.tuat.ac.jp between plasma particles and gas particles. As a result, a wall-surface jet is induced. The DBDPA has many advantages; simple structure, no mechanical moving parts and active control capability. Because of the advantages, the DBDPA is intensively studied for various aerodynamic controls: turbulent drag reduction, ${ }^{6}$ noise reduction, ${ }^{7,8)}$ and improving airfoil stall characteristics. ${ }^{2,3,9)}$ Flow separation around aerodynamic objects can be controlled by installing a DBDPA on the object surface. However, almost all studies have been conducted under laboratory-scale conditions (low speed and small-scale flow problem). DBDPAs lack sufficient flow control effect when used for mainstreams higher than $50 \mathrm{~m} / \mathrm{s}$ because the flow velocity of the jet induced is typically only several meters per second. It is indispensable to drastically improve the jet thrust in order to accelerate the practical application of DBDPAs. ${ }^{10)}$ It is believed that a

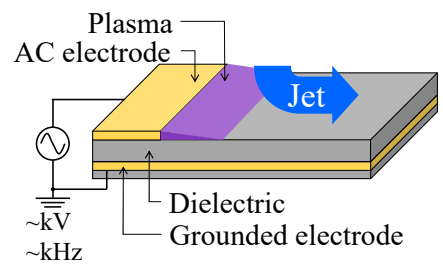

(a) DBDPA

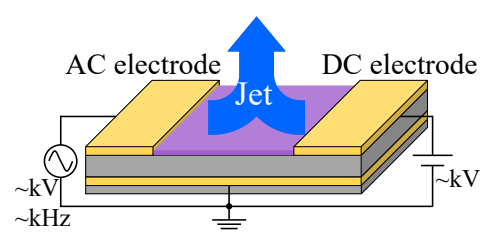

(b) TED-PA

Fig. 1. Schematics of plasma actuators. 
flow control of up to $100 \mathrm{~m} / \mathrm{s}$ in the mainstream is sufficient to enable wide-ranging applications, including aircrafts.

A tri-electrode plasma actuator (TED-PA) has been proposed to drastically improve performance. ${ }^{11-14)}$ Figure 1(b) shows the schematic configuration of the TED-PA. It has two air-exposed electrodes mounted on each side of a dielectric; a high-voltage alternating current $(\mathrm{AC})$ is applied to one electrode, and the other electrode is energized by a highvoltage direct current (DC). When a negative DC voltage is applied to the DC electrode, the TED-PA generates a discharge phenomenon called "sliding discharge"; ionized air covers the entire area between the AC and DC electrodes. ${ }^{11-13)}$ Sliding discharge was initially developed for laser pumping applications ${ }^{15)}$ and has been studied for preionization of species since many year ago. ${ }^{15-17)}$ Moreau et al. first utilized it as plasma actuator aiming to expand the plasma region of momentum exchanges, ${ }^{11)}$ and Sosa et al. reported that sliding discharge results from a combination of two discharges around the AC and DC electrodes, and the morphologies of the AC and DC discharges are largely affected by the voltages applied. ${ }^{18)}$ TED-PAs are garnering considerable interest because experimental studies have reported that they enable a significant improvement in performance to be achieved: a TED-PA can induce a jet that is 10.6 times stronger and improve electrical efficiency sixfold that of a conventional DBDPA. ${ }^{13)}$ The TED-PA is a promising solution from the viewpoint of thrust force. On the other hand, the jet induced by a TED-PA has unique behavioral characteristics: it is vertically deflected when a higher DC voltage is applied, as shown in Fig. 1(b). ${ }^{13)}$

For the practical application of TED-PAs, it is essential that application studies be conducted from the standpoint of aerodynamic control. Many application studies have been conducted for convention DBDPAs, which have provided valuable insight into flow separation control. The flow separation control mechanism realized using DBDPA changes depending on flow conditions, such as the Reynolds number. ${ }^{9,19-21)}$ Furthermore, it has been reported that the flow control effect is affected considerably by object surface layout and operating parameters such as burst frequency and ratio during burst mode: burst mode is a periodic on/off operation that enables better capability of controlling flow separation than continuous operating mode..$^{9,19-21)}$ However, there have been few application studies focused on TEDPAs, and these are indispensable in view of the unique behavioral characteristics of the jets induced.

Application studies have used computational fluid dynamics (CFD) simulations to obtain valuable results since it is possible to analyze the flow structure and impact of the plasma actuator in detail..$^{9,19-21)}$ The body force distribution generated by the actuator can be obtained using discharge plasma simulation, ${ }^{22-24)}$ and can be used in the CFD simulations as a source term of the Navier-Stokes equations. Using plasma simulations, plasma generation and detailed dynamics can be calculated by solving the continuity equations for a three-fluid coupled with Poisson's equation for an electric field in a time-marching method. This has made a consid- erable contribution to understanding the physical mechanism of plasma actuators. ${ }^{22-24)}$ However, having a high computational cost, plasma simulation is not suitable for parametric studies aimed at optimization. Accordingly, an engineering model capable of providing body force distribution using an easy, low-cost calculation process needs to be developed. Several researchers have proposed engineering models for conventional DBDPAs. ${ }^{25-27)}$ The Suzen model is an especially well-known DBDPA engineering model and has been utilized in many application studies because it can reproduce the comprehensive structure of the jet induced. ${ }^{28)}$ However, there are no engineering models for TED-PAs. In this study, in order to accelerate application studies for TED-PAs, we develop an engineering model by extending the Suzen model.

\section{DBDPA Model (Suzen Model)}

The Suzen model was proposed to easily compute the body force distribution generated by a DBDPA. ${ }^{26)}$ The body force vector is calculated using the product of the electric field vector and the charge density, as shown in Eq. (1). In the Suzen model, the electric field vector and charge density are calculated by solving two independent Poisson's equations, as shown in Eqs. (2) and (3). The Poisson's equations are numerically solved using the SOR method.

$$
\begin{gathered}
\boldsymbol{F}=\rho_{c} \boldsymbol{E} \\
\nabla \cdot\left(\varepsilon_{r} \nabla \phi\right)=0 \\
\nabla \cdot\left(\varepsilon_{r} \nabla \rho_{c}\right)=\rho_{c} / \lambda_{D}{ }^{2}
\end{gathered}
$$

Figure 2 shows the computational domains and boundary conditions. In Fig. 2, the DBDPA model and TED-PA model are shown in (a) and (b), respectively. The computational domain is divided into rectangular grid systems: grid spacing is

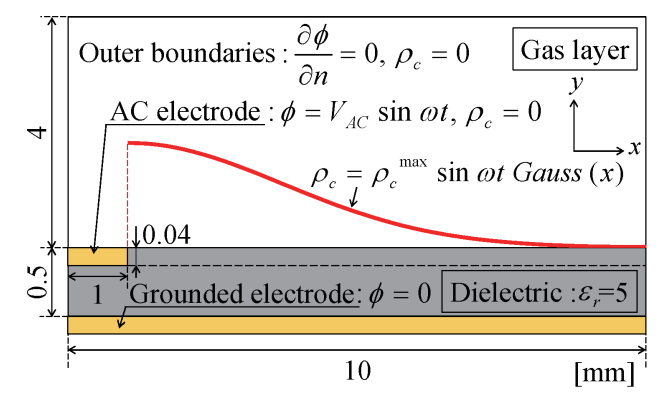

(a) DBDPA model

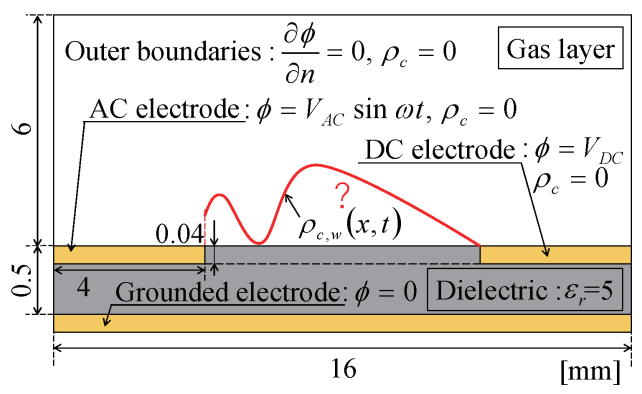

(b) TED-PA model

Fig. 2. Computational domains. 
constant at $20 \mu \mathrm{m}$. Equation (2) can be solved for the electric potential by fixing the voltage waveform applied and actuator element configuration. On the other hand, it is necessary to assume the value of Debye length and charge distribution on the dielectric surface as boundary conditions to obtain the charge density distribution from Eq. (3). In the model, the Debye length and surface charge distribution are not the physically-based parameters, but the tuning parameters to adjust the body force distribution. In particular, the surface charge distribution profile is one of the important tuning parameters governing the extent and profile of the body force. The surface charge distribution profile of the DBDPA model was proposed by Suzen et al. ${ }^{26)}$ : it is the half-Gaussian distribution having the maximum value at the edge of the AC electrode and decaying to zero at the end of the grounded electrode, and taking into account the time variation with the alternating voltage applied to the AC electrode. Although the surface charge distribution varies discontinuously on a time scale of nanoseconds and is spatially discontinuous, it is modeled to be spatially continuous and vary continuously on a time scale of alternating voltage. This assumption can be justified by considering the long time scale for fluid response. In addition, the maximum value of the surface charge distribution is also an important tuning parameter governing the magnitude of body force. Therefore, the maximum value of the surface charge distribution has to be appropriately adjusted based on experimental data. The Debye length is the tuning parameter that influences body force distribution profile. Although the Debye length is not constant on space, it is set to be a constant value $\left(\lambda_{D}=1 \mathrm{~mm}\right)$ for the model, as adopted for Suzen et al. ${ }^{28)}$ The validity of the DBDPA model proposed by Suzen et al. has been evaluated by making comparisons with experimental results, and it has been confirmed that the DBDPA model can reproduce the flow pattern and maximum flow velocity of jet induced quantitatively. ${ }^{28)}$

\section{Development of TED-PA Model}

Although the model is developed based on physical considerations, the parameters in the model are unable to be determined based on said considerations: the parameters need to be tuned artificially to reproduce the body force distribution. Therefore, to develop the TED-PA model, this study proposes how to determine TED-PA models using tuning parameters to reproduce the characteristics of body force generation. The key parameter in the TED-PA model is the surface charge distribution because it governs the profile and magnitude of the body force distribution, as with the DBDPA model. On the other hand, the Debye length is set to the same value as the DBDPA model because it was confirmed that the Debye length has little influence on body force distribution. In this study, we propose a function to provide the profile and maximum value of surface charge distribution based on the results of discharge plasma simulation, in which a three-fluid plasma model is adopted. ${ }^{22-24)}$ A simulation code developed by our research group is used, and it is confirmed that it qualitatively agrees with the experiments. ${ }^{14,23,24,29)}$ The computa-

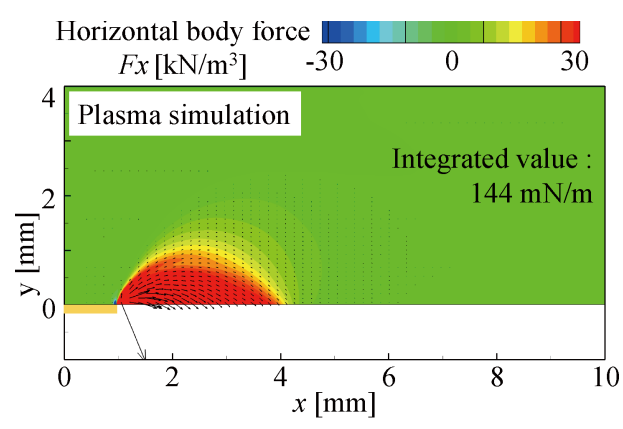

(a) Plasma simulation

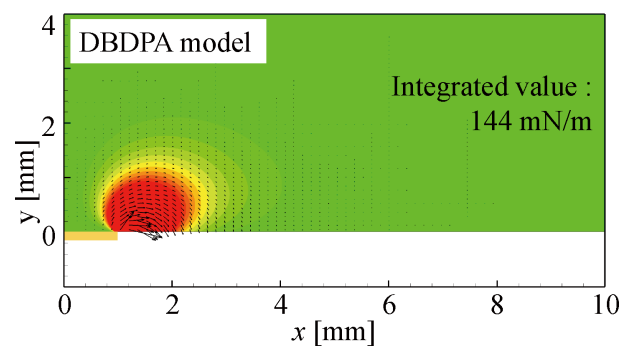

(b) DBDPA model

Fig. 3. Body force distribution of DBDPA.

tional domain for the plasma simulation of DBDPA and TED-PA is the same as that for the DBDPA and TED-PA models shown in Figs. 2(a) and 2(b), respectively.

First, we define a function to describe the surface charge distribution profile. Figure 3 shows the time-averaged distribution of the horizontal body force and body force vector generated by the DBDPA when the AC voltage amplitude applied is $28 \mathrm{kV}_{\mathrm{pp}}$ and the frequency is $10 \mathrm{kHz}$. In Fig. 3, the results of plasma simulation and the DBDPA model are shown in (a) and (b), respectively. Note that, the maximum value of the surface charge for the DBDPA model is adjusted so as to make the volume-integral value of the body force in the model correspond to that in the simulation. The comparison between Figs. 3(a) and 3(b) shows some discrepancies. In terms of the body force distribution shape, the positive body force region expands more rightward in the plasma simulation compared to that for the model. On the other hand, it does not expand leftward from the edge of the AC electrode in the simulation, while it expands leftward for the model. These discrepancies are due to simplification using the DBDPA model. In the plasma simulation, the discharge plasma evolution and dynamics are precisely simulated, and the body force is obtained self-consistently. On the other hand, the model assumes that the distribution of plasma is governed by the potential resulting from surface charge distribution, and the direction of body force is governed by the external electric field generated by the voltage applied. Although there are some discrepancies, it can be said that the model reproduces a comprehensive body force distribution structure in spite of quite simple assumptions: positive body force is generated near the edge of the AC electrode, and the positive body force region spreads rightward along the dielectric surface from the edge of the AC electrode. Therefore, the reproducibility of body force generation using 


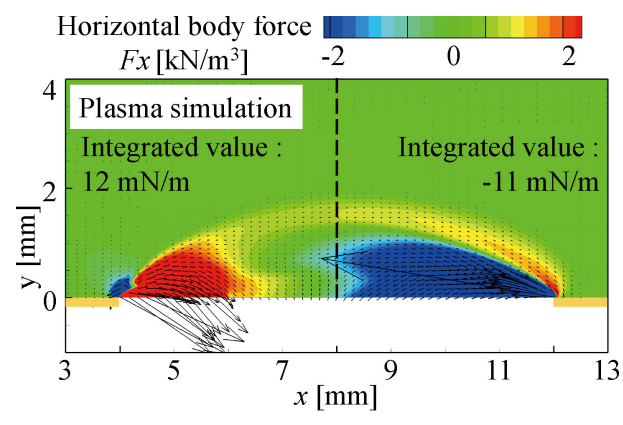

(a) Plasma simulation

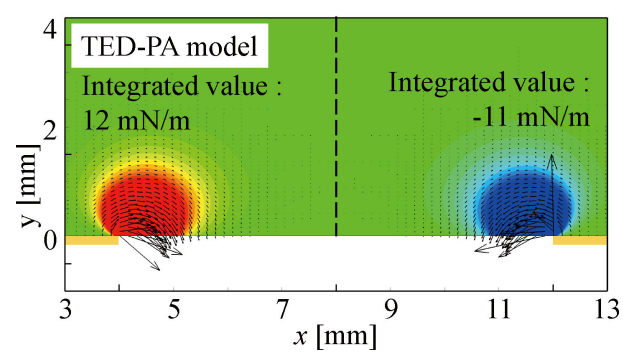

(b) TED-PA model

Fig. 4. Body force distribution of TED-PA.

the model is expected to be sufficient to reproduce the flow field induced by the DBDPA.

Figure 4(a) shows the plasma simulation results of timeaveraged horizontal body force distribution for the TEDPA with an AC voltage amplitude of $12 \mathrm{kV}_{\mathrm{pp}}$, an AC voltage frequency of $16 \mathrm{kHz}$ and a DC voltage of $10 \mathrm{kV}$. From the comparison between Fig. 3(a) and Fig. 4(a), the TED-PA generates a positive body force near the AC electrode, similar to that using the DBDPA. In addition, the TED-PA generates a negative body force near the DC electrode. The generation of positive and negative body forces results in a jet being induced perpendicular to the dielectric surface: this jet behavior was observed in previous experiments when applying a high DC voltage amplitude. ${ }^{11-13)}$ The rightward jet generated by the AC electrode and leftward jet generated by the DC electrode collide with each other, and then the collision of the two jets generates an upward deflected jet. As shown in Fig. 4(a), the body force distribution profile near the DC electrode has the same trend as that near the AC electrode: the strong body force region spreads leftward along the dielectric surface from the edge of the DC electrode. Therefore, we define the surface charge distribution profile of the TED-PA model as two half-Gaussian distributions, with distribution having the maximum value $\rho_{c}^{\max }$ at the edge of the AC and DC electrodes, which then decays to zero at the center of the electrodes. The half-Gaussian distribution on the AC electrode side takes into account the time variation with the alternating voltage applied to the AC electrode. The function to provide the surface charge distribution profile is shown in the following equation:

$$
\begin{aligned}
\rho_{c, w}(x, t)= & \rho_{c, \mathrm{AC}}^{\max }(V) \operatorname{Gauss}_{\mathrm{AC}}(x) \sin \omega t \\
& +\rho_{c, \mathrm{DC}}^{\max }(V) \operatorname{Gauss}_{\mathrm{DC}}(x)
\end{aligned}
$$

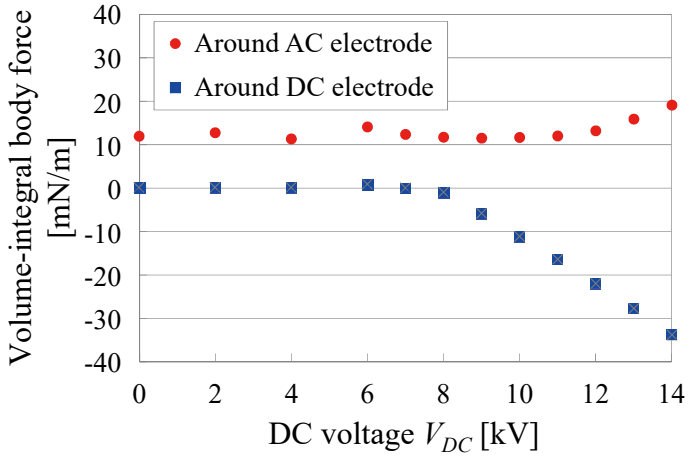

Fig. 5. Volume-integral body force versus DC voltage.

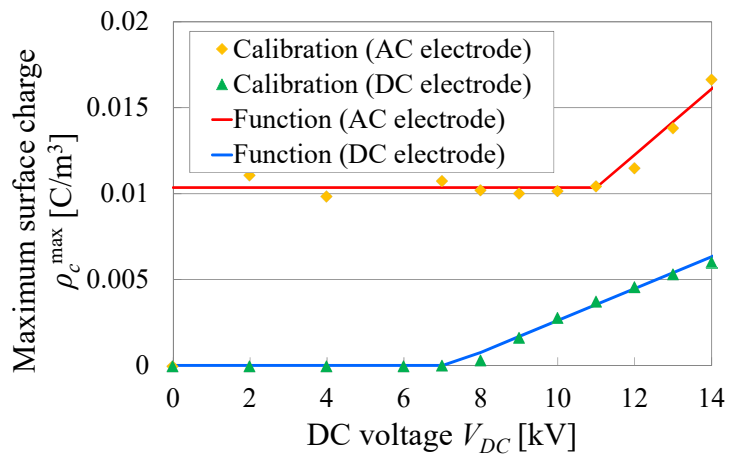

Fig. 6. Maximum values of surface charge versus DC voltage.

Here, $\operatorname{Gauss}_{\mathrm{AC}}(x)$ and $\operatorname{Gauss}_{\mathrm{DC}}(x)$ are the Gaussian functions. Each function has a maximum value $(=1)$ at the edge of the AC and DC electrodes, and decays to zero at the center of the electrode $(x=8 \mathrm{~mm})$.

Next, we define functions to provide the maximum values of the surface charge: the value at the edge of the AC electrode $\rho_{c, \mathrm{AC}}^{\max }$ and that of the DC electrode $\rho_{c, \mathrm{DC}}^{\max }$. For the TED-PA model, it is necessary to appropriately adjust $\rho_{c, \mathrm{AC}}^{\max }$ and $\rho_{c, \mathrm{DC}}^{\max }$ depending on the voltage applied in order to quantitatively reproduce the strength and deflection angle of the jet induced. In this study, we make functions to describe the voltage characteristics of $\rho_{c, \mathrm{AC}}^{\max }$ and $\rho_{c, \mathrm{DC}}^{\max }$ utilizing plasma simulation. Figure 5 shows the volume-integral values of the horizontal body force as a function of the DC voltage obtained during plasma simulation. The body force around the AC electrode is integrated over the domain $x=0-8 \mathrm{~mm}$ and $y=0-6 \mathrm{~mm}$, and the body force around the DC electrode is integrated over the domain $x=8-16 \mathrm{~mm}$ and $y=0$ $6 \mathrm{~mm}$, respectively. As shown in Fig. 5, the body force around the AC electrode increases slightly when the DC voltage becomes larger than $11 \mathrm{kV}$, and that around the DC electrode increases drastically when the DC voltage becomes larger than $7 \mathrm{kV}$. In Fig. 6, $\rho_{c, \mathrm{AC}}^{\max }$ and $\rho_{c, \mathrm{DC}}^{\max }$ are plotted against the DC voltage. They are calibrated so that the volumeintegral values of the body force for the TED-PA model match those of the simulation results. As shown in the figure, $\rho_{c, \mathrm{AC}}^{\max }$ increases when the DC voltage becomes larger than $11 \mathrm{kV}$, and $\rho_{c, \mathrm{DC}}^{\max }$ increases quasi-linearly when the DC voltage becomes larger than $7 \mathrm{kV}$. Therefore, we define $\rho_{c, \mathrm{AC}}^{\max }$ and $\rho_{c, \mathrm{DC}}^{\max }$ as functions that increase linearly with $\mathrm{DC}$ voltage 
when it exceeds a threshold value. In Fig. 6, the functions defined by providing the voltage characteristics of $\rho_{c, \mathrm{AC}}^{\max }$ and $\rho_{c, \mathrm{DC}}^{\max }$ are shown.

Figure 4(b) shows the time-averaged distribution of the horizontal body force obtained for the TED-PA model using the functions defined above. The comparison between Figs. 4(a) and 4(b) shows some discrepancies not only near the AC electrode, but also near the DC electrode. In terms of body force distribution shape, the negative body force around the DC electrode expands more leftward in the simulation compared to that for the TED-PA model. On the other hand, it does not expand rightward from the edge of the DC electrode in the simulation, while it expands rightward for the model. In terms of body force vectors, the characteristics of vertical body force generation near the DC electrode are different between the simulation and model. As discussed in the comparison between Figs. 3(a) and 3(b), these discrepancies are due to the simplification realized using the model. However, it can be said that the TED-PA model does well to reproduce a comprehensive body force distribution structure not only near the AC electrode, but also near the DC electrode. The AC electrode generates a positive body force as well as the DBDPA model and the DC electrode generates a negative body force. The negative body force region spreads leftward along the surface from the edge of the DC electrode. Moreover, it is expected that the discrepancy in vertical body force generation does not affect the comprehensive characteristics because the vertical body force is much smaller than the horizontal body force. Therefore, the TED-PA model is expected to reproduce body force distribution accurately enough to reproduce the flow field induced by the TED-PA.

\section{Reproduction of Experimental Results}

In this section, we explain how to reproduce the flow induced by an actuator manufactured in the real world using the model we developed.

The model developed utilizes the actuator element configuration: the input parameter is voltage applied and the output is body force distribution. In addition, the functions providing $\rho_{c, \mathrm{AC}}^{\max }$ and $\rho_{c, \mathrm{DC}}^{\max }$ have to be determined. The functions are governed by five parameters: $\rho_{c, \mathrm{AC}}^{\max }$ at a DC voltage of $0 \mathrm{kV}$, the threshold voltage at which $\rho_{c, \mathrm{DC}}^{\max }$ starts to increase, the threshold voltage at which $\rho_{c, \mathrm{AC}}^{\max }$ starts to increase, the slope of the $\rho_{c, \mathrm{AC}}^{\max }$ function and the slope of the $\rho_{c, \mathrm{DC}}^{\max }$ function. These parameters can be determined by comparing the horizontal and vertical thrust forces between the model and experiment at several DC voltages. In the experiment, the thrust force is the reaction force against generating the jet and is obtained by measuring the weight difference before and after actuation using an electrical balance. The thrust force is frequently used to indicate the performance of plasma actuators because it can be measured easily compared to other measurements such as flow visualization using the particle-image-velocimetry (PIV) method. In the model calculation, the thrust force is obtained by conducting fluid simulation in- corporating the body force distribution obtained using the TED-PA model.

Firstly, when the DC voltage is $0 \mathrm{kV}, \rho_{c, \mathrm{AC}}^{\max }$ can be calibrated by comparing the horizontal thrust force between the experiment and simulation. Secondly, the threshold voltage for $\rho_{c, \mathrm{DC}}^{\max }$ is from the DC voltage at which the horizontal thrust starts to decrease in the experiment. Likewise, the threshold voltage for $\rho_{c, \mathrm{AC}}^{\max }$ is from the DC voltage at which the vertical thrust appears in the experiment. Finally, the slopes of the $\rho_{c, \mathrm{AC}}^{\max }$ and $\rho_{c, \mathrm{DC}}^{\max }$ functions can be determined by finding the DC voltage at which a vertical jet is obtained; that is, the horizontal force is equal to zero. At that DC voltage, the body force strength around the AC electrode should be equal to that around the DC electrode. The body force strength around the AC electrode is characterized by $\rho_{c, \mathrm{AC}}^{\max } V_{\mathrm{AC}} / 2$ and the body force strength around the DC electrode is characterized by $\rho_{c, \mathrm{DC}}^{\max } V_{\mathrm{DC}}$. Here, $V_{\mathrm{AC}}$ is the peak voltage of the $\mathrm{AC}$ voltage and $V_{\mathrm{DC}}$ is the DC voltage when the vertical jet is obtained in the experiment. Furthermore, $1 / 2$ is a factor derived from time-averaging the alternating voltage applied to the AC electrode for one cycle. Therefore, the following relation is derived from the equilibrium of body force:

$$
\rho_{c, \mathrm{DC}}^{\max } V_{\mathrm{DC}}=\frac{1}{2} \rho_{c, \mathrm{AC}}^{\max } V_{\mathrm{AC}}
$$

$\rho_{c, \mathrm{AC}}^{\max }$ can be calibrated by comparing the vertical thrust forces of the experiment and simulation, and $\rho_{c, \mathrm{DC}}^{\max }$ can be determined by $\rho_{c, \mathrm{AC}}^{\max }$ using Eq. (5). Finally, the slopes of functions $\rho_{c, \mathrm{AC}}^{\max }$ and $\rho_{c, \mathrm{DC}}^{\max }$ can be determined by $V_{\mathrm{DC}}, \rho_{c, \mathrm{AC}}^{\max }$ and $\rho_{c, \mathrm{DC}}^{\max }$.

The results obtained using the calibrated TED-PA model are compared with the experimental results obtained by Matsuno et al. ${ }^{30)}$ The computational conditions, such as the length of electrodes, and the thickness and material of the dielectric layer in the TED-PA model are set according to the experimental conditions. ${ }^{30)}$ The AC voltage amplitude is $15.6 \mathrm{kV}_{\mathrm{pp}}$ and the frequency is $16 \mathrm{kHz}$. To obtain the flow fields and thrust force in the TED-PA model, incompressible fluid simulation is conducted. The governing equations are the incompressible Navier-Stokes equations and the continuity equation. The time-averaged body force distribution obtained using the calibrated TED-PA model is incorporated with the Navier-Stokes equations as the source term. The governing equations are solved using the marker and cell (MAC) method; the spatial derivative is evaluated using the two-order central difference method; time integration is performed using the first-order Euler method; and the Poisson's equation for pressure is solved using the SOR method in a staggered grid system. The computational setting is shown in Fig. 7. Domain (A) corresponds to the area of the TED-PA model. The flow is simulated in a much larger area than that for the TED-PA model. The same grid system is used for Domain (A) as that for the TED-PA model. Out of Domain (A), the grid spacing is gradually stretched to the simulation boundary. The grid number is 3001 in the $x$ direction and 601 in the $y$ direction. The pressure is fixed at atmos- 


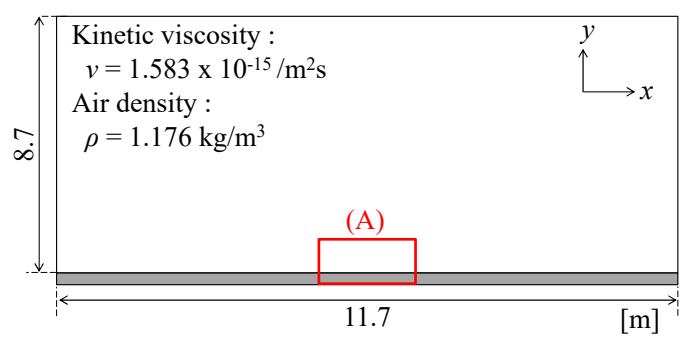

Fig. 7. Computational setting for fluid simulation.

pheric pressure $\left(=1.013 \times 10^{5} \mathrm{~Pa}\right)$ at the right, left, and top boundaries. For flow velocity, a zero-gradient boundary condition is implemented at the left, right, and top boundaries, and a nonslip wall boundary condition is implemented on the dielectric surface.

Figure 8 shows the thrust force as a function of the DC voltage applied: the horizontal and vertical components are separately plotted. Figure 9 shows the time-averaged flow field induced using the TED-PA: the flow fields are plotted for DC voltages of (a) $14 \mathrm{kV}$, (b) $16 \mathrm{kV}$, (c) $18 \mathrm{kV}$ and (d) $23 \mathrm{kV}$. In Figs. 8 and 9, the results for the TED-PA model and experiments are plotted together for comparison. In the experiment, flow visualization is conducted using PIV measurements. Figure 8 shows the TED-PA model can quantitatively reproduce the voltage characteristics of the thrust force: when the DC voltage is $0 \mathrm{kV}$, only the horizontal component is generated. When the DC voltage is increased, the vertical component increases drastically, while the horizontal component decreases with the DC voltage amplitude. Figure 9 shows that the TED-PA model can also reproduce the jet structure induced. The jet is generated not only by the AC electrode, but also by the DC electrode, and collision of the two jets generates an upward deflected jet. While the jet is inclined toward the DC electrode when the DC voltage is 14,16 and $18 \mathrm{kV}$, the jet is vertically deflected when the DC voltage is increased to $23 \mathrm{kV}$.

However, the model does not precisely correspond to the experiment in terms of vertical thrust force only in the range of 14 to $15 \mathrm{kV}$ of DC voltage, as shown in Fig. 8. In this situation, as observed in the experimental results (Fig. 9(a)), the jet induced by the AC electrode is separated by the weak jet induced by the DC electrode, resulting in the transition to turbulent flow and reattachment. Consequently, at the DC voltage in the range of 11 to $14 \mathrm{kV}$, the horizontal component decreases while the vertical thrust does not increase, as shown in Fig. 8. On the other hand, in the model, an upward deflected jet is induced, as shown in Fig. 9(a). The discrepancy is considered to be due to the two-dimensional laminar flow simulation, which is unable to reproduce the turbulent flow structure. For this reason, only in the transition regime from horizontal to upward deflected jet, the vertical thrust force in the model is larger than that in the experiment. However, the TED-PA model quantitatively reproduces the thrust force except for this regime, which implies that the TED-PA model also successfully reproduces the generation of body force quantitatively in the transition regime. Note that the

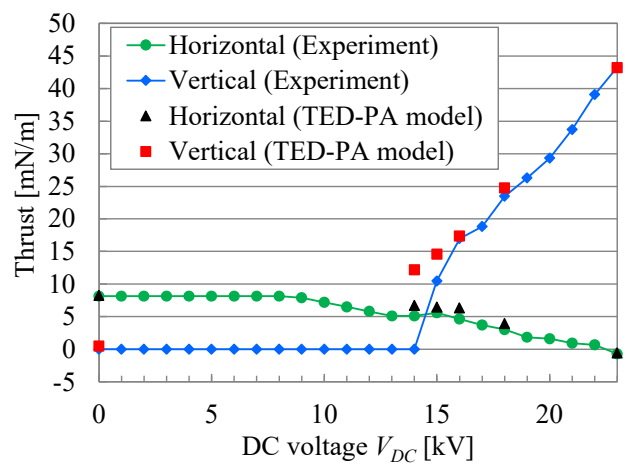

Fig. 8. Thrust versus DC voltage on TED-PA.

TED-PA model is also expected to reproduce the jet structure in the transition regime by simulating the flow field with three-dimensional fluid simulation or utilizing turbulence models.

In addition, Fig. 9 indicates that there is a considerable difference in the width of upward deflected jet between the experiment and the TED-PA model when the DC voltage is 16,18 and $23 \mathrm{kV}$. The comparison of velocity profiles for the absolute velocity at $y=20 \mathrm{~mm}$ is shown in Fig. 10 for DC voltages of (a) $16 \mathrm{kV}$, (b) $18 \mathrm{kV}$ and (c) $23 \mathrm{kV}$. As observed in Fig. 10, although the model well reproduces the $x$-position of maximum velocity within a range of $4 \mathrm{~mm}$, the width of the upward deflected jet in the TED-PA model is smaller than that in the experiment, and the maximum velocity in the model is larger than that in the experiment. In the experiment, the jet deflected upward is unstable and fluctuates over time, as shown in Fig. 11: the instantaneous flow fields obtained using PIV measurements in the case of a DC voltage of $23 \mathrm{kV}$ are plotted in the figure. The instability is seemingly due to external disturbances such as slight roughness on the dielectric surface and circulation of the flow induced in the chamber. On the other hand, the jet induced is stable and does not fluctuate in the model because the twodimensional laminar flow simulation is unable to reproduce the transition to turbulent flow and the turbulence mixing effect. Hence, the width of jet deflected upward is underestimated in the model. Note that the reproducibility of the jet width is expected to be improved by conducting threedimensional fluid simulation. Additionally, the jet width is influenced by the spatial resolution of the PIV measurements. A domain of approximately $200 \mathrm{~mm} \times 200 \mathrm{~mm}$ was taken using a camera with a resolution of $1024 \times 1000$ pixels, ${ }^{30)}$ and the vector number for the area shown in Fig. 9 was only approximately $104 \times 63$ for the experiment, while the grid number for the TED-PA model was $2500 \times 332$.

Furthermore, the inclination of the jet deflected upward seems to be slightly different for the model and experiment when the DC voltage is $23 \mathrm{kV}$, as shown in Fig. 9(d). However, the thrust force is matched perfectly, as shown in Fig. 8. This is believed to be due to the accuracy of PIV measurements, such as the spatial resolution and lack of total time for the time-averaging of instantaneous flow fields.

As discussed above, the TED-PA model quantitatively re- 


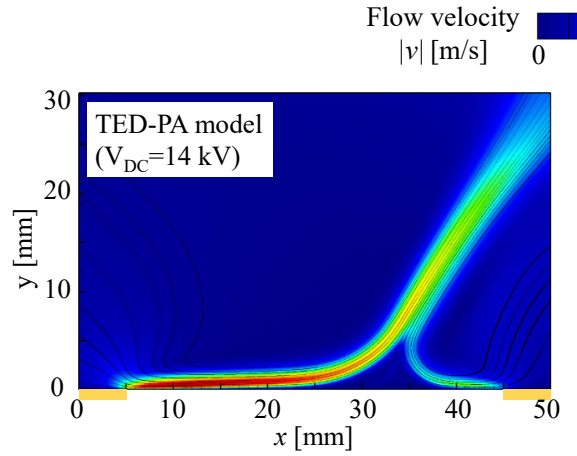

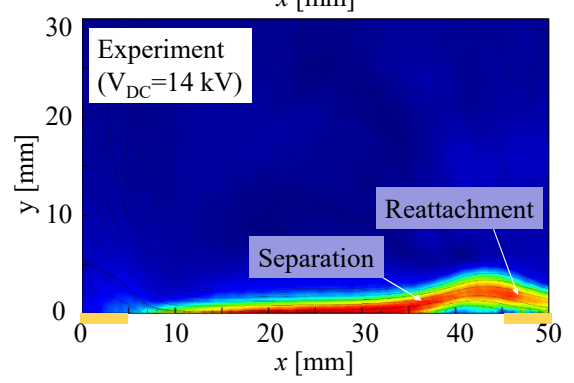

(a) $V_{\mathrm{DC}}=14 \mathrm{kV}$
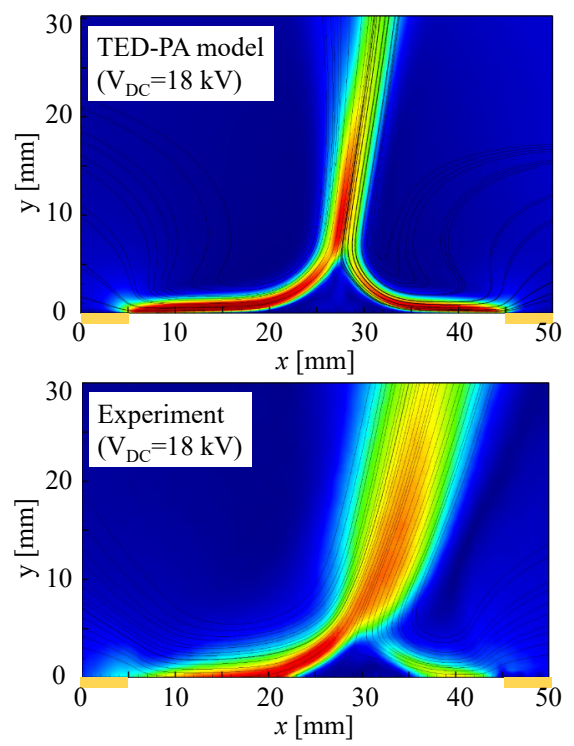

(c) $V_{\mathrm{DC}}=18 \mathrm{kV}$
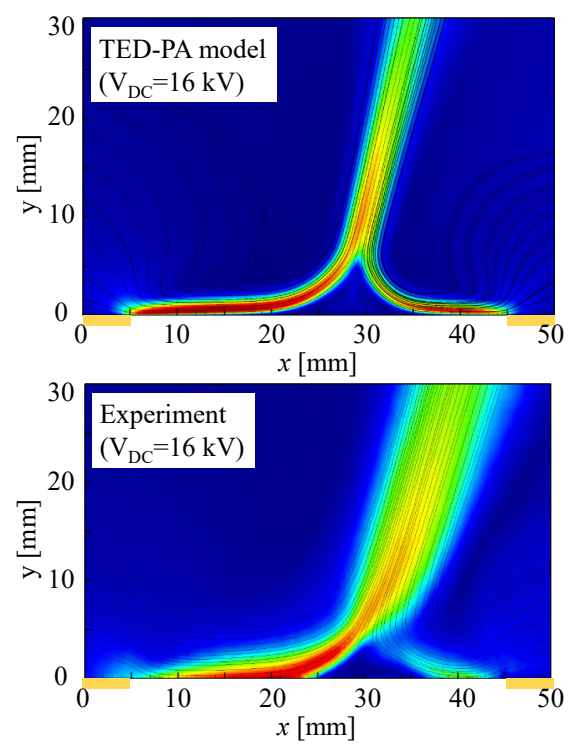

(b) $V_{\mathrm{DC}}=16 \mathrm{kV}$
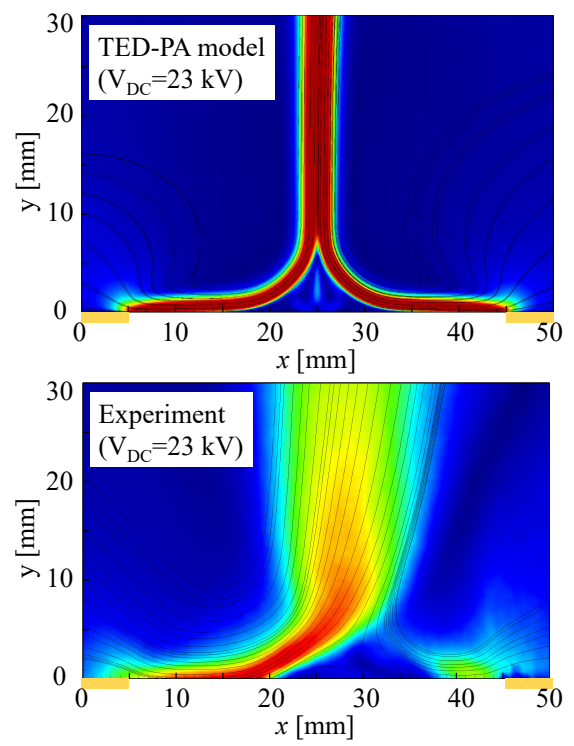

(d) $V_{\mathrm{DC}}=23 \mathrm{kV}$

Fig. 9. Time-averaged flow filed induced by the TED-PA.

produces the thrust force and a comprehensive jet structure despite the discrepancies in the flow field (i.e., vertical thrust force in the transition regime, and width and inclination of jet deflected upward), which are mainly due to fluid simulation in the current study. Therefore, the TED-PA model is expected to evaluate the flow control effect precisely since it is governed by the momentum input to the boundary layer flow.

\section{Conclusion}

In order to develop a TED-PA engineering model capable of providing body force distribution, we extended the Suzen model proposed for conventional DBDPAs. We proposed functions to provide surface charge distribution based on the results of discharge plasma simulation because surface charge distribution is the tuning parameter that governs the profile and magnitude of body force distribution.

First, we adopted two half-Gaussian functions to describe the surface charge profile: each distribution has the maximum value at the edge of the AC and DC electrodes, and it decays to zero at the center of the electrodes. Second, we defined the maximum values of the surface charge using functions of the voltage applied: the maximum values increase linearly when the DC voltage exceeds a threshold voltage, and the functions need to be calibrated by comparing the thrust force between the model and experiment at several DC voltages.

Finally, the flow fields numerically using the TED-PA model developed were compared with the experimental re- 


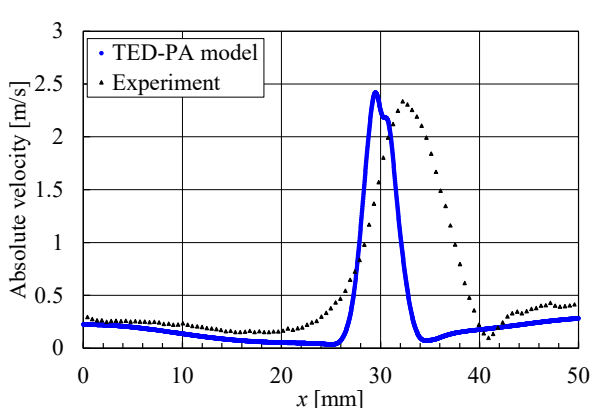

(a) $V_{\mathrm{DC}}=16 \mathrm{kV}$

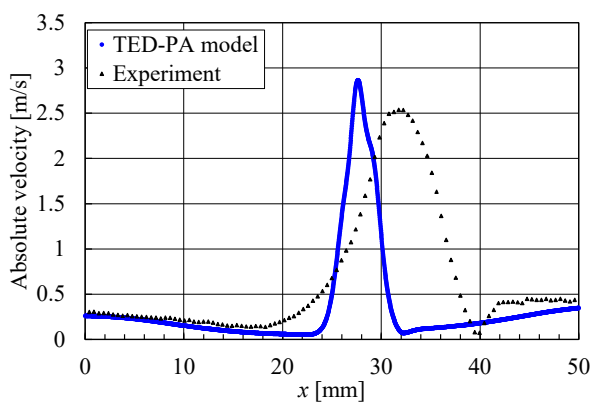

(b) $V_{\mathrm{DC}}=18 \mathrm{kV}$

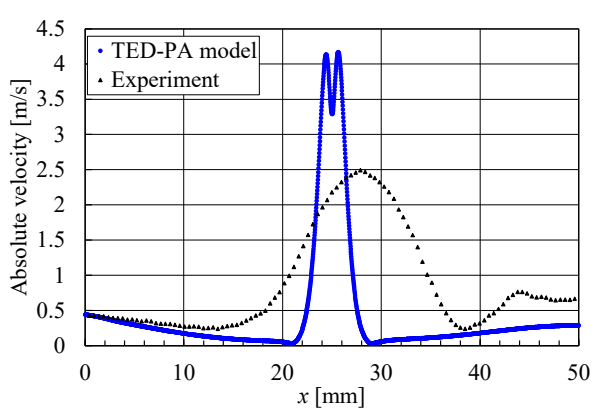

(c) $V_{\mathrm{DC}}=23 \mathrm{kV}$

Fig. 10. Absolute velocity profile at $y=20 \mathrm{~mm}$.

sults of a previous study. ${ }^{30)}$ The TED-PA model can quantitatively reproduce the voltage characteristics of thrust force and the jet structure induced. When the DC voltage exceeds the threshold value, collision of the two jets generated respectively by the AC and DC electrodes produces a jet that is deflected upward. As a result of this deflection, the thrust force vertical component begins to increase drastically as voltage amplitude increases. The inclination of the jet deflected upward, which depends on the voltage amplitude, is also successfully reproduced by the model. On the other hand, there are discrepancies in the width and maximum flow velocity of the jet deflected upward, as well as the thrust force vertical component in the transition regime from horizontal to deflected upward. This is mainly due to the two-dimensional laminar flow simulation. Therefore, the TED-PA model is expected to reproduce the jet induced (i.e., thrust force, jet structure, jet width and maximum flow velocity) and evaluate the flow control effect precisely by simulating the flow field using three-dimensional fluid simulation or a turbulence model.

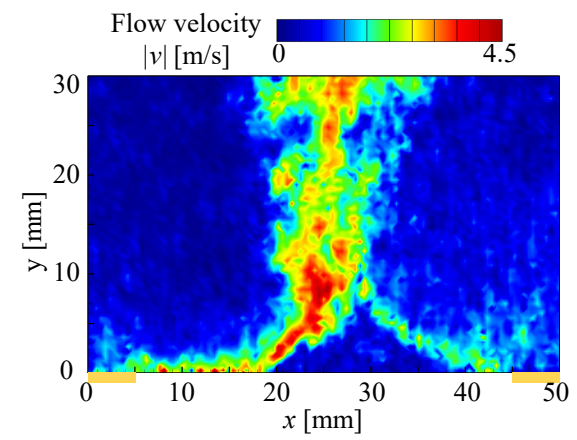

(a)

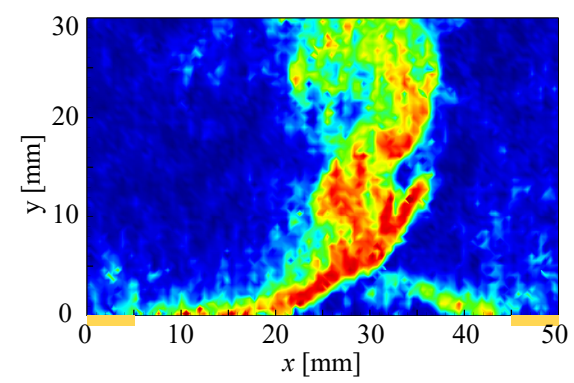

(b)

Fig. 11. Instantaneous flow field induced by the TED-PA obtained using PIV measurements.

\section{Acknowledgments}

The authors are grateful to IHI Corporation for financial support. We also thank Mr. Masaki Tamura and Ms. Asa Nakano for their valuable cooperation.

\section{References}

1) Roth, J. R., Sherman, D. M., and Wilkinson, S. P.: Electrohydrodynamic Flow Control with a Glow-Discharge Surface Plasma, AIAA J., 38 (2000), pp. 1166-1172.

2) Post, M. L. and Corke, T. C.: Separation Control on High Angle of Attack Airfoil Using Plasma Actuators, AIAA J., 42 (2004), pp. 2177-2184.

3) Huang, J., Corke, T. C., and Thomas, F. O.: Plasma Actuators for Separation Control of Low-Pressure Turbine Blades, AIAA J., 44 (2006), pp. 54-57.

4) Benard, N. and Moreau, E.: Electrical and Mechanical Characteristics of Surface AC Dielectric Barrier Discharge Plasma Actuator Applied to Air Flow Control, Exp. Fluids, 55, 1846 (2014), pp. 1-43.

5) Corke, T. C., Post, M. L., and Orlov, D. M.: SDBD Plasma Enhanced Aerodynamics: Concepts, Optimization and Applications, Progr. Aerospace Sci., 43 (2007), pp. 193-217.

6) Juke, T. N., Choi, K. J., Johnson, G. A., and Scott, S. J.: Turbulent Drag Reduction by Surface Plasma through Spanwise Flow Oscillation, AIAA Paper 2006-3693, 2006.

7) Thomas, F. O., Kozlov, A., and Corke, T. C.: Plasma Actuator for Cylinder Flow Control and Noise Reduction, AIAA J., 46 (2008), pp. 1921-1931.

8) Mitsumoji, T., Takaishi, T., Sato, Y., Ikeda, M., Seuki, T., and Fukagata, K.: A Basic Study of Aerodynamic Noise Reduction Method of a Pantograph Head Using Plasma Actuator, Quarterly Rep. RTUI, 55 (2014), pp. 184-189.

9) Fujii, K.: High-Performance Computing-Based Exploration of Flow Control with Micro Devices, Philosophical Transaction A, 372 (2014), 20130326.

10) Nishida, H., Mizuki, S., Miyazaki, I., Nonaka, S., Nonomura, T., and Inatani, Y.: Preliminary Experimental Study on Aerodynamic Charac- 
teristics Control of Slender Body Using DBD Plasma Actuator, Trans. JSASS Aerospace Technology Japan, 10, ists28 (2012), pp. Pe_97Pe_103.

11) Moreau, E., Sosa, R., and Artana, G.: Electric Wind Produced by Surface Plasma Actuators: a New Dielectric Barrier Discharge Based on a Three-Electrode Geometry, J. Phys. D Appl. Phys., 41, 11 (2008), pp. 115-204.

12) Sosa, R., Arnaud, E., Memin, E., and Artana, G.: Study of the Flow Induced by a Sliding Discharge, IEEE Trans. Dielectrics Electrical Insulation, 16 (2009), pp. 305-311.

13) Matsuno, T., Sugahara, M., Kawazoe, H., and Nishida, H.: Development of Serrated Multi-Electrode Plasma Actuators for Enhanced Force Production, AIAA Paper 2016-1691, 2016.

14) Nishida, H., Nakai, K., and Matsuno, T.: Physical Mechanism of TriElectrode Plasma Actuator with Direct-Current High Voltage, AIAA J., 55, 6 (2017), pp. 1852-1861.

15) Nakamura, K., Yukawa, N., Mochizuki, T., Horiguchi, S., and Nakaya, T.: Optimization of the Discharge Characteristics of a Laser Device Employing a Plasma Electrode, Appl. Phys. Lett., 49 (1986), pp. 1493-1495.

16) Arad, B., Gazit, Y., and Ludmirsky, A.: A Sliding Discharge Device for Producing Cylindrical Shock Waves, J. Phys. D Appl. Phys., 20 (1987), pp. 360-367.

17) Tsikrikas, G. N. and Serafetinides, A. A.: The Effect of Voltage Pulse Polarity on the Performance of a Sliding Discharge Pumped HF Laser, J. Phys. D Appl. Phys., 29 (1996), pp. 2806-2810.

18) Sosa, R., Kelly, H., Grondona, D., Marquez, A., Lago, V., and Artana, G.: Electrical and Plasma Characteristics of a Quasi-steady Sliding Discharge, J. Phys. D Appl. Phys., 41 (2008), 035202.

19) Tsubakino, D., Tanaka, Y., and Fujii, K.: Effective Layout of Plasma Actuators for a Flow Separation Control on a Wing, AIAA Paper 2007-2474, 2007.

20) Sato, M., Aono, H., Yakeno, A., Nonomura, T., Fujii, K., Okada, K., and Asada, K.: Multifactorial Effects of Operating Conditions of Dielectric-Barrier-Discharge Plasma Actuator on Laminar-SeparatedFlow Control, AIAA J., 53 (2015), pp. 2544-2559.
21) Aono, H., Sekimoto, S., Sato, M., Yakeno, A., Nonomura, T., and Fujii, K.: Computational and Experimental Analysis of Flow Structures Induced by a Plasma Actuator with Burst Modulations in Quiescent Air, Mech. Eng. J., 2 (2015), 15-00233.

22) Boeuf, J. P., Lagmich, Y., and Pitchford, L. C.: Contribution of Positive and Negative Ions to the Electrohydrodynamic Force in a Dielectric Barrier Discharge Plasma Actuator Operating in Air, J. Appl. Phys., 106 (2009), 023115.

23) Nishida, H. and Abe, T.: Validation Study of Numerical Simulation of Discharge Plasma on DBD Plasma Actuator, AIAA Paper 2011-3913, 2011.

24) Nishida, H., Nonomura, T., and Abe, T.: Three-Dimensional Simulations of Discharge Plasma Evolution on a Dielectric Barrier Discharge Plasma Actuator, J. Appl. Phys., 115 (2014), 013302.

25) Shyy, W., Jayaraman, B., and Andersson, A.: Modeling of Glow Discharge-Induced Fluid Dynamics, J. Appl. Phys., 92 (2002), pp. 6434-6443.

26) Suzen, Y. B., Huang, P. G., Jacob, J. D., and Ashpis, D. E.: Numerical Simulations of Plasma Based Flow Control Applications, AIAA Paper 2005-4633, 2005.

27) Mertz, B. E. and Corke, T. C.: Time-Dependent Dielectric Barrier Discharge Plasma Actuator Modeling, AIAA Paper 2009-1083, 2009.

28) Suzen, Y. B., Huang, P. G., and Ashpis, D. E.: Numerical Simulations of Flow Separation Control in Low-Pressure Turbines using Plasma Actuators, AIAA Paper 2007-937, 2007.

29) Nakai, K., Nakano, A., and Nishida, H.: Validation Study of Threefluid Plasma Modeling of Dielectric Barrier Discharge for Plasma Actuator, AIAA Paper 2018-0937, 2018.

30) Matsuno, T., Sugahara, M., Koyama, J., Fujita, N., Yamada, G., and Kawazoe, H.: Vectored Jet Control for Trielectrode Plasma Actuator with Serrated Electrode, Trans. JSASS Aerospace Technology Japan, 14, ists30 (2016), pp. Pe_55-Pe_61.

Naofumi Ohnishi Associate Editor 